\title{
Clinical Management of Acute Portal/Mesenteric Vein Thrombosis
}

\author{
Sven A. Lang ${ }^{a}$ Martin Loss ${ }^{a}$ Walter A. Wohlgemuth ${ }^{b}$ Hans J. Schlitt ${ }^{a}$ \\ a Department of Surgery, University Hospital Regensburg, Regensburg, Germany, \\ ${ }^{b}$ Department of Radiology, University Hospital Regensburg, Regensburg, Germany
}

\section{Keywords}

Portal/mesenteric vein thrombosis - Recanalization . Intervention · Surgery

\section{Summary}

Background: Acute thrombosis of the portal vein (PV) and/ or the mesenteric vein (MV) is a rare but potentially lifethreatening disease. A multitude of risk factors for acute portal vein thrombosis (PVT)/mesenteric vein thrombosis (MVT) have been identified, including liver cirrhosis, malignancy, coagulation disorders, intra-abdominal infection/inflammation, and postoperative condition. Methods: This article analyses the treatment options for acute PVT/MVT. Results: Initially, the clinical management should identify patients with an intra-abdominal focus requiring immediate surgical intervention (e.g. bowel ischaemia). Subsequently, emphasis is placed on the recanalization of the PV/MV or at least the prevention of thrombus extension to avoid longterm complications of portal hypertension. Several therapeutic options are currently available, including anticoagulation therapy, local/systemic thrombolysis, interventional or surgical thrombectomy, and a combination of these procedures. Due to the lack of prospective randomized studies, a comparison between these therapeutic approaches regarding the efficacy of PV/MV recanalization is difficult, if not impossible. Conclusion: In patients with acute PVT/MVT, an individualized treatment based on the clinical presentation, the underlying disease, the extent of the thrombosis, and the patients' comorbidities is mandatory. Therefore, these patients should be considered for an interdisciplinary therapy in specialized centres with the option to utilise all therapeutic approaches currently available.

\author{
Schlüsselwörter \\ Pfortader-/Mesenterialvenenthrombose . \\ Rekanalisation · Intervention · Chirurgie
}

\section{Zusammenfassung}

Hintergrund: Die akute Thrombose der Portalvene (PV) und/ oder der Mesenterialvene(n) (MV) ist eine seltene, jedoch potenziell lebensbedrohliche Erkrankung. Eine Vielzahl von Risikofaktoren wie Leberzirrhose, maligne Tumoren, Gerinnungsstörungen, intraabdominelle Infektionen/Entzündungen und postoperativer Zustand konnte mit der akuten Portalvenen-/Mesenterialvenenthrombose (PVT/MVT) in Zusammenhang gebracht werden. Methoden: In der vorliegenden Arbeit werden die Behandlungsoptionen bei akuter PVT/MVT analysiert. Ergebnisse: Das Management der akuten PVT/MVT sollte zunächst Patienten identifizieren, die aufgrund eines intraabdominellen Fokus eine umgehende chirurgische Therapie benötigen (z.B. Darmischämie). Ziel der weiteren Behandlung sollte dann eine Rekanalisation der PV/MV oder zumindest eine Verhinderung der Thromboseausbreitung sein, um Langzeitkomplikationen der portalen Hypertonie zu verhindern. Verschiedene therapeutische Optionen, z.B. die therapeutische Antikoagulation, lokale/systemische Lyse, interventionelle oder chirurgische Thrombektomie, bzw. deren Kombination stehen zur Verfügung. Aufgrund fehlender prospektiv-randomisierter Studien ist jedoch ein Vergleich hinsichtlich der Effektivität der einzelnen Therapieverfahren schwierig, wenn nicht gar unmöglich. Schlussfolgerung: Eine individualisierte Therapie unter Berücksichtigung des klinischen Erscheinungsbilds, der zugrunde liegenden Erkrankung, der Ausdehnung der Thrombose und der Komorbiditäten des Patienten ist aus unserer Sicht dennoch erforderlich. Daher sollten Patienten mit akuter PVT/MVT einer interdisziplinären Behandlung an spezialisierten Zentren, die alle derzeit vorhandenen Therapieoptionen anbieten können, zugeführt werden.

\section{KARGER \\ Fax +497614520714

\section{(C) 2014 S. Karger GmbH, Freiburg}

1662-6664/14/0306-0394\$39.50/0
PD Dr. med. Sven Arke Lang

Department of Surgery

University of Regensburg Medical Center

Franz-Josef-Strauß-Allee 11, 93042 Regensburg, Germany

sven.lang@ukr.de 


\section{Introduction}

Portal vein thrombosis (PVT) is defined as a complete or partial thrombosis of the main portal trunk that may also include the right and/or left intrahepatic portal branch. Furthermore, PVT may extend to the mesenteric (MV) and splenic veins, then being classified as thrombosis of the portal venous system. Some authors consider PVT to be acute if symptoms develop within 60 days prior to diagnosis although this is not a generally accepted definition $[1,2]$. In fact, others define acute PVT as symptoms occurring within 7 days prior to diagnosis and subacute PVT if symptoms last longer than 7 days $[3,4]$. The discrimination from chronic PVT is usually made according to signs of portal hypertension such as cavernous transformation of the portal vein (PV), development of collaterals/varices, and significant splenomegaly.

\section{Symptoms of Acute PVT/MVT}

Diagnosis of an acute PVT/mesenteric vein thrombosis (MVT) based on clinical findings is often challenging. Patients may be asymptomatic or may present with unspecific abdominal pain. In some cases, PVT or MVT is incidentally found during abdominal imaging that was performed for other reasons. In contrast, patients with PVT/MVT may also suffer from severe abdominal pain, gastrointestinal bleeding, vomiting, and diarrhoea. In particular, when the PVT extends to the mesenteric of splenic veins, an acute abdomen including bowel congestion, signs of ischaemia, and features of sepsis such as shock or multiorgan failure may occur. In patients with liver cirrhosis, acute decompensation may be a sign of acute PVT.

\section{Diagnostic Work-Up for Acute PVT/MVT}

The first step in the diagnostic work-up is a detailed evaluation of the patient's medical history. In particular, a history of thromboembolism, liver cirrhosis, coagulation disorders, malignancies (e.g. hepatocellular carcinoma (HCC), pancreatic cancer), intra-abdominal surgical procedures (e.g. splenectomy, colectomy), inflammatory lesion (e.g. pancreatitis, appendicitis), abdominal trauma, or use of oral contraceptives have been associated with acute PVT or MVT. So far, there are no specific laboratory tests to identify an acute PVT/ MVT. Sometimes an unspecific elevation of leukocytes can be recognized. Nonetheless, in cases suspect for PVT/MVT, the initial laboratory tests should include at least infection parameters as well as liver, kidney, and basic coagulation values. The next step usually is an abdominal colour Doppler ultrasound (CDUS) which has a sensitivity and specificity of about $80-100 \%$ to detect PVT in most studies but might be limited in MVT (reviewed in [5]). Contrast-enhanced ultrasound
(CEUS) is an additional diagnostic tool that may be useful in patients with low portal velocity [6]. Results from CDUS (and CEUS), however, are dependent on the experiences of the operator. Moreover, information about the extent of the thrombosis and the underlying disease may be limited. Therefore, further diagnostic work-up with contrast-enhanced computed tomography (CECT) or magnetic resonance imaging (MRI) is often needed. In particular, patients presenting with suspicious clinical findings in the abdominal examination should be considered for CT or MRI to detect intra-abdominal foci that require immediate surgical treatment (reviewed in [5]). In contrast to MRI, CT is usually faster in providing results with fewer artefacts due to patient movement and is available $24 \mathrm{~h} /$ day. Therefore, we suggest a CECT scan with portal and arterial phase as the imaging technique of choice.

\section{Initial Hospital Treatment of Acute PVT/MVT}

Therapy of patients with acute PVT or MVT strongly depends on their clinical presentation. In general, when the diagnosis of acute PVT/MVT is made, an immediate start of anticoagulation therapy is required. We prefer to use unfractionated heparin, aiming at a partial thromboplastin time (PTT) between 60 and $80 \mathrm{~s}$ due to the easier peri-interventional handling. Alternatively, low-molecular-weight heparin (LMWH) may be used. In order to prevent extension of the thrombosis, anticoagulation therapy should also be initiated if further therapeutic procedures such as interventional recanalization or even surgery are planned. In addition, we recommend an immediate initiation of broad-spectrum antibiotic therapy since bacteraemia, e.g. from Bacteroides fragilis, is associated with PVT [7]. Sufficient pain control, replacement of fluids, and electrolytes upon acute presentation are compulsory. Naturally, patients presenting with shock or even multiorgan failure require specific therapy including treatment on the intensive care unit (ICU). The subsequent goal in patients with acute PVT/MVT is to detect clinical situations requiring immediate surgical intervention. In the case of suspicious findings during the abdominal examination or in the CT scan, e.g. signs of bowel ischaemia or perforation, an explorative laparotomy to remove the focus is mandatory. This includes potential bowel resection and, depending on the extent of the thrombosis and the intraoperative situation, surgical thrombectomy of the PV/MV. In addition, we tend to insert a lysis catheter via a MV in these patients (fig. 1). A second-look laparotomy to reassess the bowel perfusion is performed either after $24 \mathrm{~h}$ or on demand.

Most patients do not present with an acute abdomen requiring immediate surgery. In these patients, therapeutic strategies in acute PVT/MVT strongly depend on the underlying disease. In general, three main groups can be defined: patients with liver cirrhosis, with malignancies, and those unrelated to cirrhosis or malignancies. The primary therapeutic aim in these patients with acute PVT/MVT is to achieve reca- 


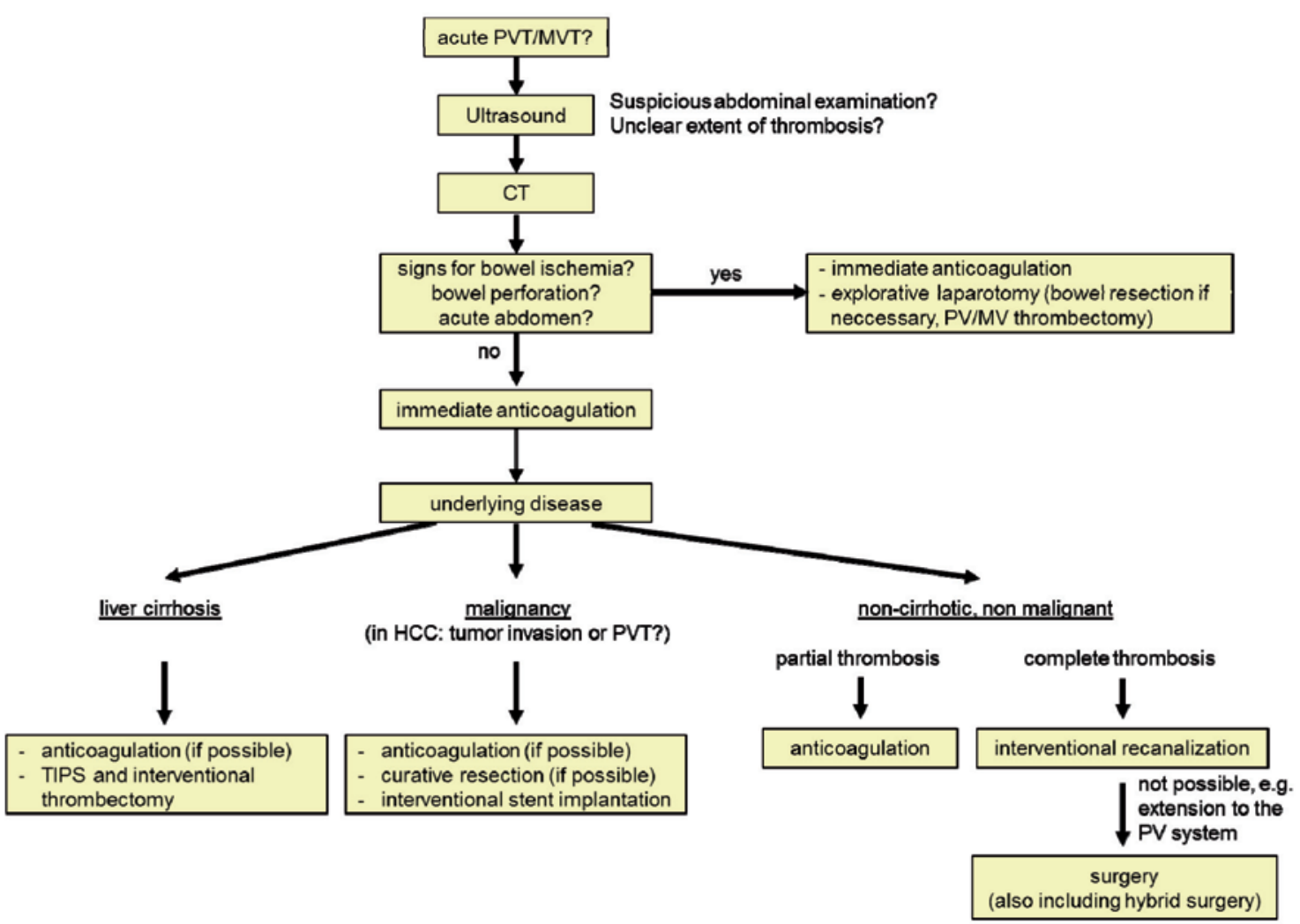

nalization of the PV/MV or at least to prevent thrombus extension to avoid a complication of chronic PVT/MVT, e.g. the development of varices, haemorrhage, or ascites. Therapeutic options for local recanalization include anticoagulation, regional or systemic thrombolysis, thrombectomy, or a combination of these techniques. Due to the lack of prospective randomized trials, a direct comparison between these opportunities is difficult, if not impossible.

\section{Therapy in Acute PVT}

\section{PVT in Liver Cirrhosis}

PVT is a frequent problem in patients with advanced liver cirrhosis. The definition of the 'age' of a PVT may be difficult in these patients since common features of chronic PVT (collateral circulation, portal hypertension) are also a part of liver disease [8]. In addition, PVT in liver cirrhosis might be associated with $\mathrm{HCC}$; therefore, an intensive search for $\mathrm{HCC}$ has to be performed. However, the presence of acute PVT in patients with liver cirrhosis clearly increases the risk of variceal bleeding and may also lead to a life-threatening emergency when it extents to the MVs (reviewed in [9]). So far, no randomized controlled trials have been performed to evaluate the best treatment strategy for acute PVT in liver cirrhosis. Several reports indicate that anticoagulation provides a good chance for recanalization even in concomitant liver cirrhosis [10-12]. In particular, a complete or partial recanalization rate of $30-60 \%$ has been reported for either vitamin $\mathrm{K}$ antagonists or the use of LMWH [9-11]. Although reports are promising, only very limited patient numbers were included; therefore, a general recommendation is critical. One might be aware of the potential problems of anticoagulation therapy in patients with liver cirrhosis such as aggravation of variceal bleeding, problems with international normalized ratio (INR) monitoring with vitamin $\mathrm{K}$ antagonists, and accumulation of LMWH in patients with impaired kidney function. Nonetheless, whenever possible the use of anticoagulation therapy should be initiated even in patients with concomitant liver cirrhosis.

Furthermore, interventional recanalization might be considered in patients with liver cirrhosis. In particular, if decompensation of liver cirrhosis is the clinical sign of acute PVT, the placement of a transjugular intrahepatic portosystemic shunt (TIPS) in combination with anticoagulation therapy and/or thrombectomy provides a good chance for recanalization, recompensation, and reduction of the complications of portal hypertension [12]. This is of particular importance for patients on the waiting list for liver transplantation. Although PVT is usually not considered as a contraindication for transplantation, pre-existing PVT is an independent prognostic marker for posttransplant survival and increases the risk for recurrent thrombosis and re-transplantation [13, 14]. Therefore, interventional PV recanalization might also help to improve post-transplant outcome. Finally, if interventional recanalization via TIPS is not possible, surgical portocaval shunt procedures remain an option in selected patients. In summary, anticoagulation in combination with interventional recanalization is our therapy of choice in patients with acute PVT in liver cirrhosis (fig. 1). 


\section{PVT in Malignancies}

Malignancies leading to acute PVT are mainly located in the hepato-pancreato-biliary system, and therapeutic strategies have to take the potentially limited prognosis into consideration. Moreover, differentiation of tumour infiltration into PV branches and a 'real' PVT might be difficult especially in patients suffering from HCC. In the case of a resectable disease, surgical therapy including PV resection and reconstruction is the treatment of choice. A recent analysis by Chok et al. [15] showed that resection of the PV bifurcation and even tumour thrombectomy might be performed with acceptable results in patients with HCC although not all PVTs were acute in this publication. Resection of the $\mathrm{PV}$ in pancreatic cancer is rarely performed because of PVT but rather due to tumour infiltration or compression. However, most patients with acute PVT and malignant disease present in an irresectable stage. In the absence of contraindications such as bleeding from the tumour, anticoagulation therapy should be initiated as the treatment of choice. In symptomatic PVT or impending closure of the PV, interventional treatment, e.g. stent implantation, might be considered [16] (fig. 1).

\section{PVT Unrelated to Cirrhosis or Malignancies}

A multitude of factors may lead to PVT in patients without cirrhosis or malignancies. In particular, diseases and conditions such as inflammation (e.g. pancreatitis), infections (e.g. B. fragilis), surgical intervention (e.g. splenectomy, liver transplantation), coagulation disorders (e.g. prothrombin gene mutation, factor V Leiden mutation), oral contraceptive use, or blunt abdominal trauma have been associated with acute PVT. To date, anticoagulation is the commonly chosen treatment mostly due to concerns over complications such as bleeding. Results from a prospective multicentre study using heparin followed by oral anticoagulation with vitamin $\mathrm{K}$ antagonists (INR between 2 and 3 ) showed a recanalization rate of the main portal trunk and its left or right branch in $39 \%$ [17]. This is in line with earlier results that were summarized by Hall et al. [18], showing a complete and partial recanalization rate of 38.3 and $14 \%$, respectively, in the patients upon anticoagulation. In patients with symptomatic acute PVT but without signs of bowel ischaemia, more aggressive therapeutic intervention might be required. One option is the administration of local or systemic thrombolytic therapy. Several agents (recombinant tissue-type plasminogen activator (rt-PA), urokinase, streptokinase) and routes of administration (intravenous, via superior mesenteric artery, or via transhepatic or transjugular application to the PV) have been reported [19, 20]. A summary of 13 articles showed $40 \%$ complete and $45 \%$ partial recanalization of the PV upon thrombolysis although four of these reports also included local thrombectomy [18]. However, these reports may suffer from a strong selection bias since thrombolytic therapy was initiated mostly after anticoagulation treatment had failed. Complications, in particular bleeding, are described in up to $60 \%$ of the patients [21].

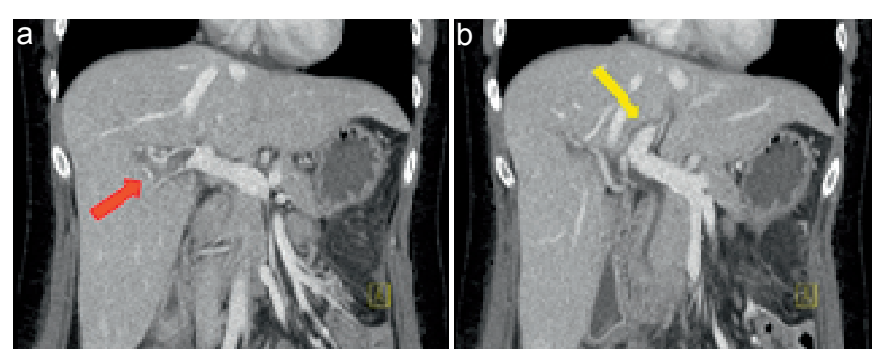

Fig. 2. a Intrahepatic thrombosis of the right portal branch after laparoscopic colectomy (red arrow). b The left portal branch is still patent (yellow arrow). Therapeutic anticoagulation was initiated.

Mechanical thrombectomy via a transhepatic or transjugular approach is another therapeutic option which is often applied in combination with local thrombolysis. It has the advantage of rapid removal of the thrombus in acute PVT. Initial recanalization rates are promising although the local vascular trauma may promote recurrent thrombosis [18, 22-24]. Surgical thrombectomy is performed only if the patient undergoes laparotomy for acute abdomen or intestinal ischaemia.

Being faced with the long-term complications of portal hypertension, we prefer a more aggressive strategy including interventional recanalization and also applying surgery in selected patients with PVT unrelated to cirrhosis or malignancy. In general, systemic anticoagulation therapy is applied to asymptomatic patients with partial thrombosis (fig. 2). In the case of symptomatic thrombosis, an interventional therapy (thrombectomy and thrombolysis) is performed. If the latter is not possible in symptomatic patients or any sign for bowel ischaemia exists, we prefer an explorative laparotomy including intraoperative thrombectomy and local lysis therapy (fig. 1).

\section{Mesenteric Vein Thrombosis}

The clinical presentation of patients with MVT is similar to that of PVT. Furthermore, the risk factors for the development of MVT are almost the same as for PVT, including prothrombotic states, thrombophilia, malignancies, intra-abdominal infections, and postoperative condition. Abdominal CECT is the diagnostic tool of choice. Similar to the management of acute PVT, evidence of bowel ischaemia, perforation, or peritonitis in MVT should lead to immediate surgical exploration. In addition, unfractionated heparin should be administered as soon as the diagnosis is made in order to prevent an extension of the thrombosis. Intraoperative assessment of bowel viability is mandatory, and evaluation of the patency of the superior mesenteric vein (SMV) is necessary. If the SMV is occluded, thrombectomy should be performed, and local thrombolysis can be undertaken via a transmesenterically inserted catheter (fig. 3). Second-look laparotomy for the reassessment of bowel viability is optional. In patients without acute abdomen upon initial presentation, an immedi- 


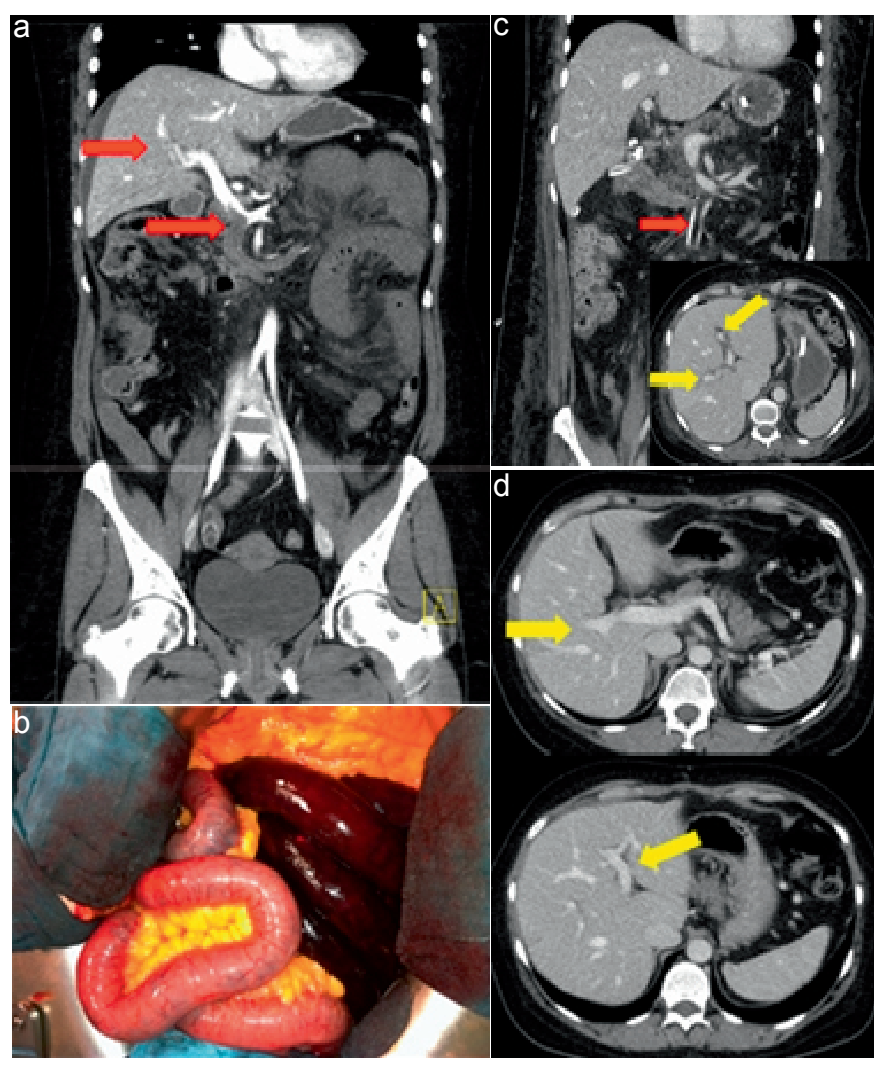

Fig. 3. Patient with acute MVT presenting with acute abdomen and bleeding from the proximal jejunum. a CT scan shows the MVT (red arrow) and partial thrombosis of the right intrahepatic portal branch. b Intraoperative evaluation of the bowel showed the need for bowel resection due to haemorrhage infarction of the proximal jejunum. In addition, a lysis catheter was inserted into the mesenteric vein. c CT scan 2 days after the initial operation showed the lysis catheter in the MV (red arrow) and confirmed patency of both intrahepatic portal branches (yellow arrows). d Patency of both intrahepatic portal branches at follow-up examination after 6 months (yellow arrows).

ate initiation of anticoagulation therapy is also recommended. Interventional thrombectomy and thrombolysis might be considered depending on the extent of thrombosis and the clinical presentation. However, evidence for the efficacy of thrombectomy and thrombolysis is limited to case reports and small retrospective series [21]. Finally, close clinical monitoring of patients with MVT, including assessment of symptoms, control of laboratory parameters, and, if necessary, repeated CT scans, is mandatory to identify those developing secondary bowel infarction.

\section{Occlusion of the Portal Venous System}

PVT and MVT can occur alone or as a combination of both. The latter is of particular importance since it increases the risk for bowel ischaemia. Therapeutic options used by most clinicians are basically the same as for PVT and MVT. We have recently described a novel strategy for patients with
Table 1. Coagulation disorders associated with acute PVT/ MVT (summarized in $[1,3,19,27])$

$$
[1,3,19,27])
$$

\section{Inherited \\ Protein S deficiency \\ Protein C deficiency \\ Antithrombin III deficiency \\ Prothrombin 20210 gene mutation \\ Factor V Leiden mutation}

acute PVT encompassing the intrahepatic branches and the SMV [4]. This includes a surgical thrombectomy via infrapancreatic venotomy of the SMV followed by intraoperative antegrade and retrograde radiologic thrombectomy via the same approach. In the case of remnant thrombotic material, local thrombolysis can be administered via a catheter placed in a MV. Follow-up after a median of 9 months showed perfusion of the main portal trunk and at least one intrahepatic branch in 4 out of 5 patients. Although this strategy is associated with significant acute complications, it remains an option for patients with thrombosis of the portal venous system not being accessible for interventional recanalization.

\section{Diagnostics for Coagulation Disorders}

The initial diagnostic efforts should also be focused on the identification of local disease such as cirrhosis, malignancies, and abdominal sepsis as a cause for acute PVT or MVT. However, acute thrombosis of the PV/MV is often multifactorial, and identification of thrombophilic conditions might have therapeutic consequences for the duration of anticoagulation even in the presence of a local factor. A multitude of thrombophilic and prothrombotic conditions have been described in the literature (table 1). Among them, factor V Leiden mutations and myeloproliferative disorders (MPD) are most commonly associated with PVT/MVT. In particular, presence of the JAK2 mutation JAK2V617F has an odds ratio exceeding 50 for acute PVT; therefore, PVT may be the first manifestation of MPD [25]. In patients with cirrhosis, prothrombin gene mutation (G20210A) has often been reported although its role in acute PVT without cirrhosis is less clear [26]. Similarly, protein $\mathrm{S}$, protein $\mathrm{C}$, and antithrombin III deficiency have been reported in PVT/MVT but their role remains unclear since serum levels might also be altered by liver injury in acute PVT/MVT [19]. Hence, the timing of diagnostics for coagulation disorders is important. Mutation analyses can be in- 
itiated immediately after the diagnosis of acute PVT/MVT is made, whereas other analyses, e.g. for protein S/C deficiency, should be performed at a certain interval after the initial thrombotic event. Although these tests are very cost-intensive, we perform a complete search for coagulation disorders in all patients with PVT/MVT without underlying cirrhosis or malignancy. Nonetheless, due to the complexity of the topic, a close cooperation with specialists in haemostaseology is highly recommended.

\section{Duration of Anticoagulation and Follow-Up}

Irrespective of the underlying disease and the chosen therapy, anticoagulation treatment should be administered to all patients after acute PVT/MVT. In fact, recanalization of the PV may occur up to 6 months after the initial event. Furthermore, re-thrombosis is a serious problem even after complete $\mathrm{PV} / \mathrm{MV}$ recanalization. However, there is currently no consensus on the duration and medication of anticoagulation. In general, 3-6 months of anticoagulation therapy is proposed by most authors $[1,19]$. Whether this also holds true for patients with liver cirrhosis is not clear so far [9]. In the case of hypercoagulopathy, a lifelong anticoagulation is usually recommended. Anticoagulation therapy during the hospital stay is mostly performed with intravenous unfractionated heparin or
LMWH. Subsequent transition to oral vitamin K antagonists with a target INR of $2-3$ is described in most studies [1, 3]. Timing and duration of follow-up examinations are also controversial. We tend to see the patients either in the surgical or in the specialized coagulation outpatient clinic every 3 months for up to 1 year at least. Depending on the localization and the extent of the thrombosis, CDUS (or CEUS) is used to assess patency. In the case of unclear results or recurrent symptoms, a CECT is performed.

\section{Conclusion}

Management of acute PVT or MVT (or both) is complex due to the lack of evidence-based therapeutic algorithms. A close cooperation between surgery, internal medicine, and radiology is important not only to prevent potentially lethal consequences of acute PVT/MVT but also to avoid the complications of chronic PVT such as portal hypertension and variceal haemorrhage. In conclusion, patients with acute PVT/ MVT should be treated in specialized centres.

\section{Disclosure Statement}

The authors declare no conflicts of interest.

\section{References}

1 Parikh S, Shah R, Kapoor P: Portal vein thrombosis. Am J Med 2010;123:111-119.

$\checkmark 2$ Ponziani FR, Zocco MA, Campanale C, Rinninella E, Tortora A, Di Maurizio L, Bombardieri G, De Cristofaro R, De Gaetano AM, Landolfi R, Gasbarrini A: Portal vein thrombosis: insight into physiopathology, diagnosis, and treatment. World J Gastroenterol 2010;16:143-155.

$\checkmark 3$ Chawla Y, Duseja A, Dhiman RK: Review article: The modern management of portal vein thrombosis. Aliment Pharmacol Ther 2009;30:881-894.

4 Loss M, Lang SA, Uller W, Wohlgemuth WA, Schlitt HJ: Combined surgical and interventional therapy of acute portal vein thrombosis without cirrhosis: a new effective hybrid approach for recanalization of the portal venous system. J Am Coll Surg 2014;218:e79-86.

5 Berzigotti A, Garcia-Criado A, Darnell A, GarciaPagan JC: Imaging in clinical decision-making for portal vein thrombosis. Nat Rev Gastroenterol Hepatol 2014;11:308-316.

6 Claudon M, Dietrich CF, Choi BI, et al: Guidelines and good clinical practice recommendations for contrast enhanced ultrasound (CEUS) in the liver - update 2012: a WFUMB-EFSUMB initiative in cooperation with representatives of AFSUMB, AIUM, ASUM, FLAUS and ICUS. Ultraschall Med 2013;34:11-29.

7 Ni YH, Wang NC, Peng MY, Chou YY, Chang FY: Bacteroides fragilis bacteremia associated with portal vein and superior mesentery vein thrombosis secondary to antithrombin III and protein C deficiency: a case report. J Microbiol Immunol Infect 2002;35:255-258.
8 Tsochatzis EA, Senzolo M, Germani G, Gatt A, Burroughs AK: Systematic review: portal vein thrombosis in cirrhosis. Aliment Pharmacol Ther 2010;31:366-374.

-9 Kinjo N, Kawanaka H, Akahoshi T, Matsumoto Y, Kamori M, Nagao Y, Hashimoto N, Uehara H, Tomikawa M, Shirabe K, Maehara Y: Portal vein thrombosis in liver cirrhosis. World J Hepatol 2014; 6:64-71.

10 Amitrano L, Guardascione MA, Menchise A, Martino R, Scaglione M, Giovine S, Romano L, Balzano A: Safety and efficacy of anticoagulation therapy with low molecular weight heparin for portal vein thrombosis in patients with liver cirrhosis. J Clin Gastroenterol 2010;44:448-451.

11 Delgado MG, Seijo S, Yepes I, Achecar L, Catalina MV, Garcia-Criado A, Abraldes JG, de la Pena J, Banares R, Albillos A, Bosch J, Garcia-Pagan JC: Efficacy and safety of anticoagulation on patients with cirrhosis and portal vein thrombosis. Clin Gastroenterol Hepatol 2012;10:776-783.

12 Senzolo M, T MS, Rossetto V, Burra P, Cillo U, Boccagni P, Gasparini D, Miotto D, Simioni P, Tsochatzis E, Burroughs AK: Prospective evaluation of anticoagulation and transjugular intrahepatic portosystemic shunt for the management of portal vein thrombosis in cirrhosis. Liver Int 2012; 32:919-927.

13 Doenecke A, Tsui TY, Zuelke C, Scherer MN, Schnitzbauer AA, Schlitt HJ, Obed A: Pre-existent portal vein thrombosis in liver transplantation: influence of pre-operative disease severity. Clin Transplant 2010;24:48-55.
14 Rodriguez-Castro KI, Porte RJ, Nadal E, Germani G, Burra P, Senzolo M: Management of nonneoplastic portal vein thrombosis in the setting of liver transplantation: a systematic review. Transplantation 2012;94:1145-1153.

15 Chok KS, Cheung TT, Chan SC, Poon RT, Fan ST, Lo CM: Surgical outcomes in hepatocellular carcinoma patients with portal vein tumor thrombosis. World J Surg 2014;38:490-496.

16 Novellas S, Denys A, Bize P, Brunner P, Motamedi JP, Gugenheim J, Caroli FX, Chevallier P: Palliative portal vein stent placement in malignant and symptomatic extrinsic portal vein stenosis or occlusion. Cardiovasc Intervent Radiol 2009;32:462-470.

17 Plessier A, Darwish-Murad S, Hernandez-Guerra M, Consigny Y, Fabris F, Trebicka J, Heller J, Morard I, Lasser L, Langlet P, Denninger MH, Vidaud D, Condat B, Hadengue A, Primignani M, Garcia-Pagan JC, Janssen HL, Valla D: Acute portal vein thrombosis unrelated to cirrhosis: a prospective multicenter follow-up study. Hepatology 2010;51:210-218.

18 Hall TC, Garcea G, Metcalfe M, Bilku D, Dennison AR: Management of acute non-cirrhotic and non-malignant portal vein thrombosis: a systematic review. World J Surg 2011;35:2510-2520.

19 Handa P, Crowther M, Douketis JD: Portal vein thrombosis: a clinician-oriented and practical review. Clin App Thromb Hemost 2013;20:498-506.

20 Liu FY, Wang MQ, Fan QS, Duan F, Wang ZJ, Song $P$ : Interventional treatment for symptomatic acutesubacute portal and superior mesenteric vein thrombosis. World J Gastroenterol 2009;15:5028-5034. 
21 Hollingshead M, Burke CT, Mauro MA, Weeks SM, Dixon RG, Jaques PF: Transcatheter thrombolytic therapy for acute mesenteric and portal vein thrombosis. J Vasc Interv Radiol 2005;16:651-661.

22 Jun KW, Kim MH, Park KM, Chun HJ, Hong KC, Jeon YS, Cho SG, Kim JY: Mechanical thrombectomy-assisted thrombolysis for acute symptomatic portal and superior mesenteric venous thrombosis. Ann Surg Treat Res 2014;86:334-341.

23 Oguzkurt P, Tercan F, Ince E, Ezer SS, Hicsonmez A: Percutaneous treatment of portal vein thrombosis in a child who has undergone splenectomy. J Pediatr Surg 2008;43:e29-32.
24 Uflacker R: Applications of percutaneous mechanical thrombectomy in transjugular intrahepatic portosystemic shunt and portal vein thrombosis. Tech Vasc Interv Radiol 2003;6:59-69.

25 Dentali F, Squizzato A, Brivio L, Appio L, Campiotti L, Crowther M, Grandi AM, Ageno W: JAK2V617F mutation for the early diagnosis of $\mathrm{Ph}$-myeloproliferative neoplasms in patients with venous thromboembolism: a meta-analysis. Blood 2009;113:5617-5623.
26 Dentali F, Galli M, Gianni M, Ageno W: Inherited thrombophilic abnormalities and risk of portal vein thrombosis. A meta-analysis. Thromb Haemost 2008;99:675-682.

27 Singal AK, Kamath PS, Tefferi A: Mesenteric venous thrombosis. Mayo Clin Proc 2013;88:285-294. 